\title{
Numerical Study of the Flow Within a Vanned Volute of a Mixed Flow Turbine
}

\author{
Ahmed Ketata, Zied Driss \\ Laboratory of Electro-Mechanic Systems (LASEM), National School of Engineers of Sfax (ENIS), University of Sfax, Sfax, Tunisia \\ Email address: \\ ketata.ahmed.enib@gmail.com (A. Ketata), zied.driss@enis.tn (Z. Driss) \\ To cite this article: \\ Ahmed Ketata, Zied Driss. Numerical Study of the Flow Within a Vanned Volute of a Mixed Flow Turbine. International Journal of Fluid \\ Mechanics \& Thermal Sciences. Vol. 3, No. 5, 2017, pp. 46-51. doi: 10.11648/j.ijfmts.20170305.11
}

Received: October 10, 2017; Accepted: October 23, 2017; Published: November 25, 2017

\begin{abstract}
A turbocharger is commonly used to get more available output power for internal combustion engines. The radial turbine recovers the engine exhaust gas energy and converts it into rotational energy. The turbocharger turbine consists mostly in a radial or mixed flow impeller and a volute. The turbocharger volute transforms a part of the engine exhaust gas energy into a kinetic energy and guides the movement towards the rotor inducer at a suitable flow angle. The main purpose of this paper is to study the flow structure within a vanned volute of a mixed flow turbine. For this end, numerical simulations are conducted by solving the Navier-Stokes equations using the commercial Computational Fluid dynamiX (CFX) package and including a finite volume discretization method. The reasonable agreement found between experimental and numerical results of the turbine performance confirms the accuracy of the numerical model. The distributions of the pressure, the velocity, and the turbulence characteristics are numerically obtained in this analysis. The results showed that the fluid flow within the turbine volute is highly turbulent. Moreover, a significant pressure damp has been recorded within the volute vane which leads to a low-pressure flow at the rotor entry. Also, it has been shown that the flow direction considerably turns from the radial direction to the tangential one across the volute casing.
\end{abstract}

Keywords: CFD, Turbulence, Mixed-Flow Turbine, Turbocharger, Performance, Mass Flow Rate, Efficiency

\section{Introduction}

Car manufacturers embed boosting systems to internal combustion engines in order to minimize the engines exhaust emissions by means of the downsizing technics. The turbine which is an important component of a turbocharger consists fundamentally of a volute and a rotor. The function of the volute is to covert a proportion of the engine exhaust gas energy and to drive the flow to the rotor inlet at appropriate conditions. The volute can be vaneless, vanned or equipped with a nozzle ring. The volute casing must be carefully chosen in purpose to provide a uniform flow at the desired angle enabling better turbine performance. Several anterior works are developed to optimize the volute design under steady and pulsating conditions. This model presents a significant effect on both the turbine performance and the discharge flow angle distribution. Lymberopoulos et al. [1] performed a quasi-three-dimensional solution based on the Euler equation to analyze the flow within single and twin entry volutes. Their results showed that the cross-section design has a substantial impact on the turbine performance. Gu et al. [2] compared the compressible and incompressible volute designs for a radial inflow turbine using computational fluid dynamics and theoretical free vortex models. Their study indicated that the flow angle distortion is mainly due to occurring wake and recirculation of the flow at the volute tongue. Barnard and Benson [3] confirmed that the volute design affects significantly the turbine performance. According to their results, the variation of the turbine performance was found be up to $1.5 \%$. Chen et al. $[4,5]$ used a quasi-steady inverse method to compare the internal flow field between trapezoidal and round cross-sectional shapes. Their results showed that the cross-sectional shape did not present a detriment effect on several flow parameters such as the absolute flow angle and the Mach number. Romagnoli et al. [6] developed a mean line model to predict the aerodynamic performance for nozzleless and nozzled volutes for a mixed flow turbine. The confrontation between their mean-line model results and their test data shows a good agreement. Furthermore, their model allows getting a breakdown analysis of the occurring losses 
within the mixed flow turbine. Yang et al. [7, 8] studied the effect of the cross-section shape for a nozzleless volute of a radial turbine under pulsating conditions. They reported that the mean efficiency, as well as the mass flow rate, significantly depends on the cross-sectional shape. Suhrmann et al. [9] conducted numerical simulations to understand the effect of the tongue geometrical parameter on the full turbine stage performance under steady conditions. Their results showed higher efficiency which can be achieved by choosing a small tongue radius combined with a small angular position. Abidat el al. [10] developed a numerical study to capture the flow filed inside a radial and a mixed flow turbine volutes. Their numerical results indicated a complex flow structure at the tongue downstream. Besides, they reported that the structure of the flow leads to a non-uniformity generation at the volute exit circumference.

As a literature review, it is clear that the volute casing and cross sections design presents a significant effect on the turbine efficiency. Most of previous works which investigated on the flow fields within the turbine volute reported the occurrence of a secondary flow generation. The presence of this secondary flow leads to the aerodynamic losses and then, to the deterioration of the turbine performance. The present paper aims to present our numerical model in order to capture the flow patterns inside a vanned volute of a turbocharger mixed flow turbine under steady conditions. Eventually, a radial based method is used to design the quasi-trapezoidal shape of the volute cross sections. Equally, the numerical methods and the meshing are presented. To confirm our numerical model accuracy, the computed and experimental turbine performances are confronted showing a reasonable agreement. Based on our numerical solution, the distribution of the pressure, the velocities, and the velocity streamlines are computed at different planes to analysis the flow behavior and to localize the expected occurring secondary flow within the turbine volute. In addition, the distribution of the vorticity, the turbulent kinetic energy, its dissipation rate and the turbulent viscosity are presented and discussed in order to characterize the turbulent flow.

\section{Geometrical Arrangement}

For turbocharger applications, the mixed flow turbine comes to be more employed instead of the radial inflow turbine. The mixed flow rotor can be seen as a cross design between radial and axial configurations. Its design allows a non-zero inlet blade angle conserving the radial fiber elements rule. Furthermore, the mixed flow turbine works efficiently at lower velocity ratios compared to the radial turbine.

The turbine investigated in this application consists of a mixed flow impeller and a vanned volute. The volute is firstly a commercial twin entry vaneless one. In these conditions, Rajoo [11] modified this volute to acquire a nozzle ring and then, it becomes a vanned volute with $140 \mathrm{~mm}$ of the inlet vane diameter. In this application, we are interested in the nozzleless configuration of the volute. Figure 1.a shows the developed geometrical model of the present volute. The volute cross sections have a quasi-trapezoidal shape, and the radius based method is used to draw these cross-sections. Its centroid throat radius is about $100 \mathrm{~mm}$. Furthermore, the ratio of its throat area to its throat radius is about 33 . The rotor of the turbine which consists of 12 blades is early designed and tested by Abidat [12]. The mean inlet diameter of the rotor is about $83.6 \mathrm{~mm}$. However, it exit diameter is about $26.43 \mathrm{~mm}$. The rotor length is equal to $40 \mathrm{~mm}$. More details about the turbine rotor design are available in the literature [12]. Figure 1.b illustrates the full assembly of the computational domain.

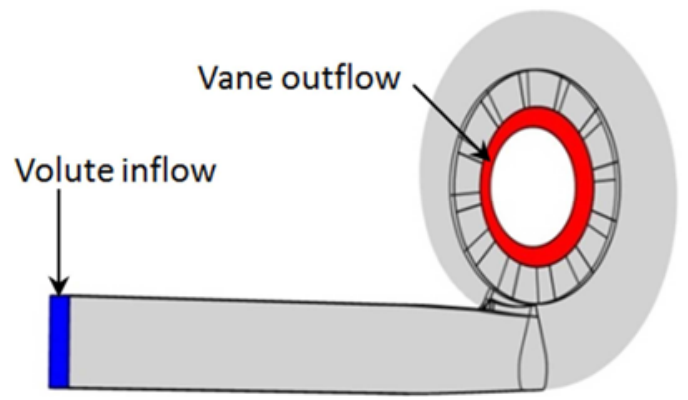

(a) The vanned volute.

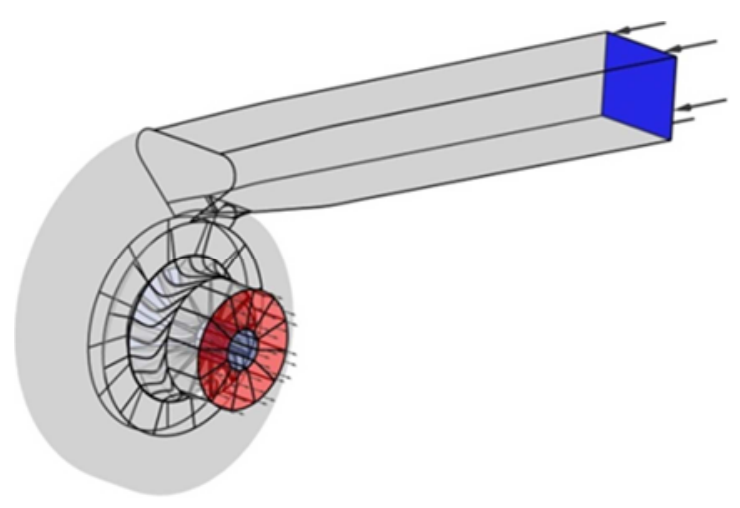

(b) Full computational domain.

Figure 1. 3D model of the full computational domain.

\section{Numerical Method}

The numerical solutions are obtained using the Ansys CFX 17.0 which is a commercial computational fluid dynamics solver. The numerical simulations consist of an implicit resolution of the Reynolds averaged Navier-Stokes equations of mass, momentum, and energy conservation including a finite volume method for the discretization. The standard $\mathrm{k}-\varepsilon$ turbulence model is chosen to solve the additional terms due to the Reynolds decomposition. Good results are achieved by means of this turbulence model in many anterior aerodynamic works $[13,14]$. Then, the Ansys software is used for the mesh generation. In fact, the computational domain is divided into 329619 tetrahedral cells and 106070 nodes (figure 2 ). To get a suitable non-dimensional wall distance that matches with our turbulence model, ten prism layers are applied to the near wall regions of the computational domain. Figure 3 shows a three-dimensional view of our developed unstructured meshing for the mixed flow turbine. In addition, the approach of the Multiple Reference Frame (MRF) used previously by 
Lam et al. [15] is chosen to model the rotating domain. As boundary conditions, a total pressure of $213996 \mathrm{~Pa}$ and a total temperature of $343 \mathrm{~K}$ are fixed at the full turbine stage inlet boundary. Eventually, a static pressure is defined at the turbine outlet. However, the rotational speed of the turbine shaft is set to be $59783.4 \mathrm{rpm}$. Furthermore, all flow parameters are set to take null values for the initialization of the computational domain.

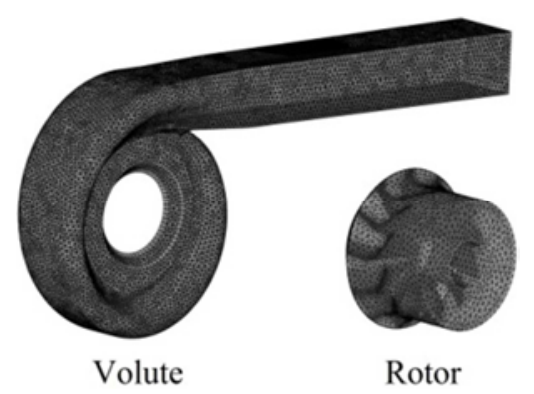

Figure 2. Meshing.

\section{Comparison with the Test Data}

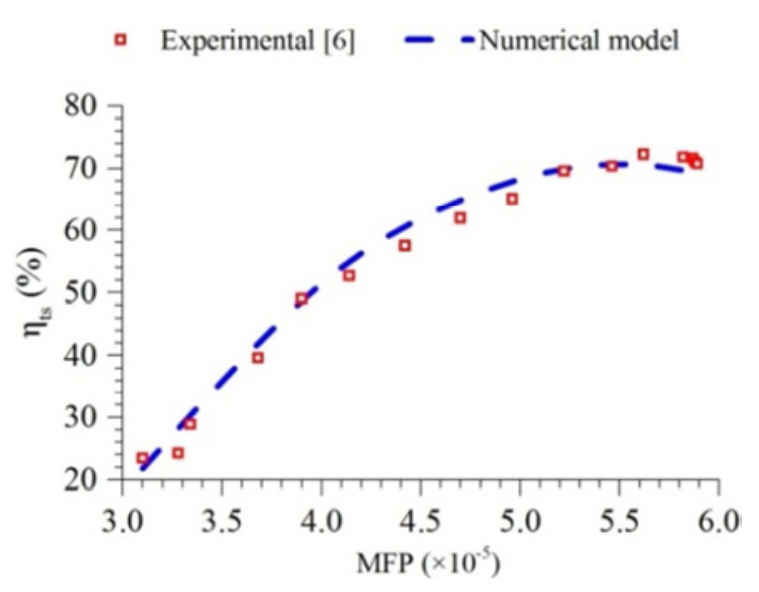

Figure 3. Superposition of the numerical result to test data.

This section attempts to verify our numerical model validity by comparing numerical results to the experimental data, collected by Romagnoli et al. [6] at the rotational speed of $59783.4 \mathrm{rpm}$. For this, the total to static isentropic efficiency is plotted against the mass flow parameter. Figure 3 presents the confrontation of the computed results coming out from our numerical computations to the test data. From these results, it has been observed that the numerical distribution of the turbine efficiency matches well with the experimental data. The standard deviation between the numerical and experimental results of the efficiency is found to be $2.37 \%$. However, the standard deviation for the mass flow parameter is about $4.69 \%$.

These values of the standard deviation seem to be in an acceptable range. For this, our numerical model can be considered valid.

\section{Results and Discussion}

As shown in figure 4, the mid-span transverse plane is chosen to visualize the different flow parameters: the velocity, the pressure, the vorticity, the turbulent kinetic energy, its dissipation rate and the turbulent viscosity. All these parameters are computed for 2.968 of the turbine expansion ratio and $59783.4 \mathrm{rpm}$ of the rotational speed.

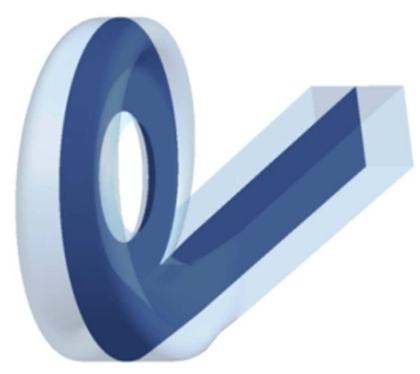

Figure 4. Mid-span transverse visualization plane.

\subsection{Velocity Vectors}

Figure 5 shows the distribution of the velocity superposed to its vectors at the mid-span transverse plane. From these results, it has been observed that the velocity magnitude remains approximately constant throughout the volute duct. Furthermore, the direction of the flow is found to be uniform. Downstream of the inlet throat of the volute, the flow turns gradually to follow the volute scroll. This fact is due to the increase of the tangential component of the velocity magnitude. In addition, it has been noted a slight decline of the velocity magnitude at the near wall zones. However, an important improvement of the velocity magnitude has been recorded across the volute vane.

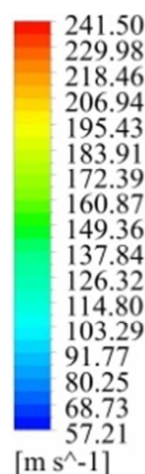

$\left[\mathrm{m} \mathrm{s}^{\wedge}-1\right]$

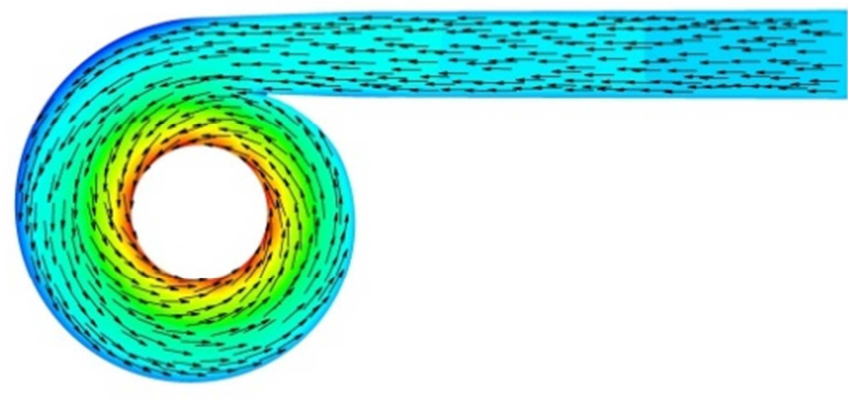

Figure 5. Velocity vectors. 
In fact, higher values of the velocity magnitude occur at the volute vane exit. For example, the velocity magnitude at the volute inlet throat is found to be about $115 \mathrm{~m} . \mathrm{s}^{-1}$, and then it gets an upward trend across the scroll to be finally about 240 m.s- 1 at the vane exit. Thus, the flow is accelerated throughout the volute and leaves the volute with a high tangential component of the velocity.

\subsection{Static Pressure}

Figure 6 presents the distribution of the static pressure at the mid-span transverse plane. From these results, it has been noted that the static pressure remains nearly at the same level across the volute inlet duct. Downstream of the volute inlet throat, the pressure drops progressively throughout the volute scroll, and it achieves its lowest values at the volute vane exit. For example, the static pressure is found to be around 201457 $\mathrm{Pa}$ at the volute inlet throat, and then, it decreases sharply to be about $154376 \mathrm{~Pa}$ at the vane exit. However, it has been observed that the zone near the wall keeps approximately the same value of the static pressure which is about $200000 \mathrm{~Pa}$ as that recorded at the inlet duct.

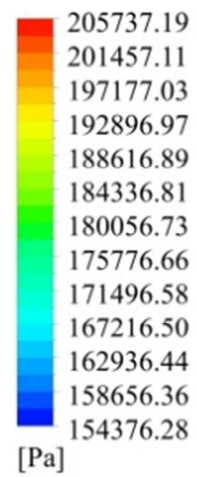

$[\mathrm{Pa}]$

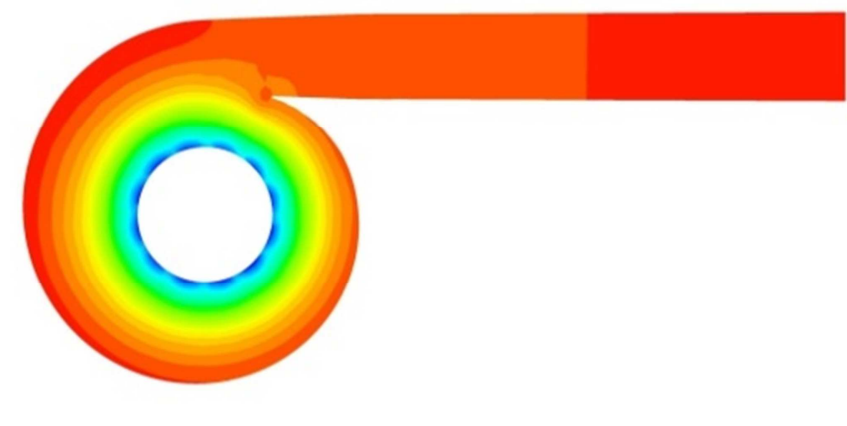

Figure 6. Distribution of the pressure.

\subsection{Vorticity}

Figure 7 shows the distribution of the vorticity at the mid-span transverse plane.

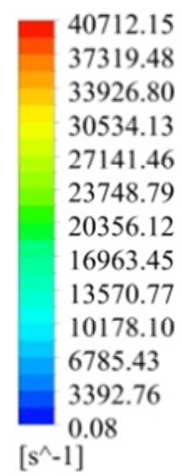

37319.48

33926.80

30534.13

27141.46

6963.45

3570.77

0178.10

6785.43

$\left[\mathrm{s}^{\wedge}-1\right]$

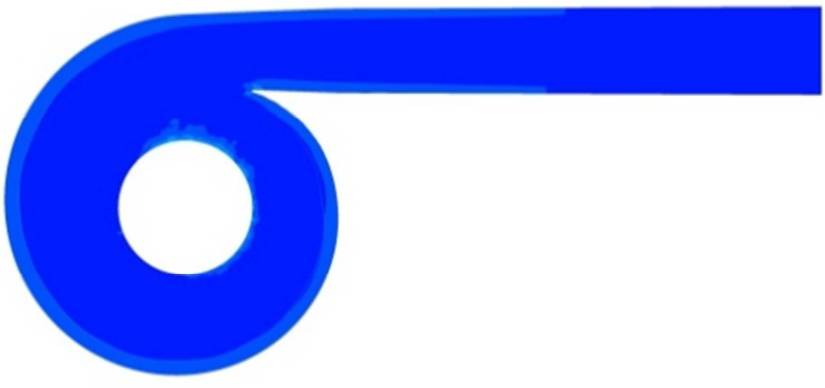

Figure 7. Distribution of the vorticity.
From these results, it can be seen that the vorticity stills in the same level with a very weak value from the volute inlet duct to the vane exit except the region near the wall. At this region, a wake zone characteristic of the maximum values of the vorticity emerges. Furthermore, a slight increase of the vorticity is equally recorded at the near tongue region of the vane exit. These areas characteristic of the maximum values of the vorticity are limited regarding the full domain of the volute and then can be neglected. Thus, the flow within the volute can be considered globally uniform.

\subsection{Turbulent Kinetic Energy}

Figure 8 shows the distribution of the turbulent kinetic energy at the mid-span transverse plane. From these results, it has been observed that the turbulent kinetic energy presents weak values at the volute inlet duct. Across the volute scroll, it has been found that the turbulent kinetic energy gets an upward trend. However, a wake characteristic of the maximum values of the turbulent kinetic energy is recorded in the near wall regions. This zone extends to the tongue upstream in which the turbulent kinetic energy is found to be around $46 \mathrm{~m}^{2} \cdot \mathrm{s}^{-2}$. Thus, the tongue seems to be a production seat of turbulent kinetic energy. In addition, from the vane inlet zone, the turbulent kinetic energy growths significantly across the volute vane until the vane exit which presents maximum values of the turbulent kinetic energy, around 70 $\mathrm{m}^{2} \cdot \mathrm{s}^{-2}$, for an azimuth angle between $-10^{\circ}$ to $100^{\circ}$. 

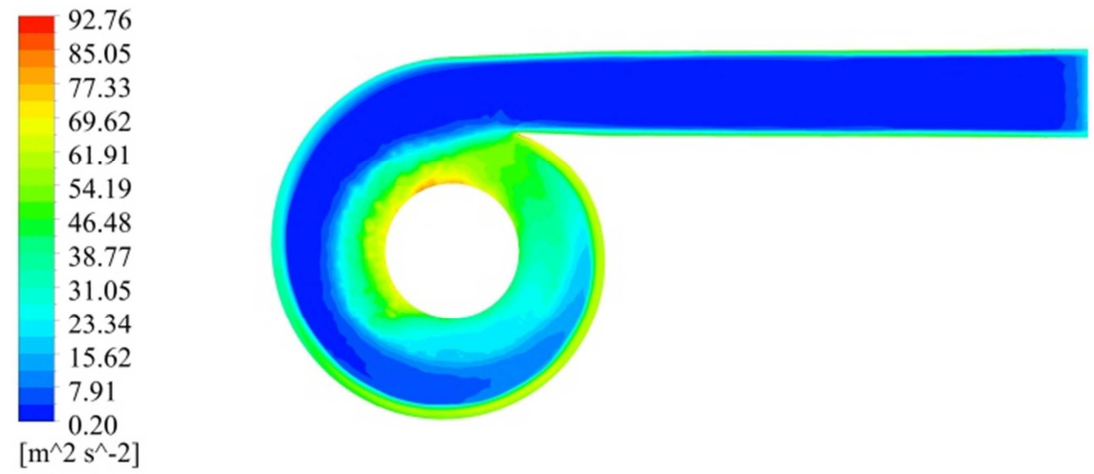

Figure 8. Distribution of the turbulent kinetic energy.

\subsection{Dissipation Rate of the Turbulent Kinetic Energy}

Figure 9 illustrates the distribution of the dissipation rate of the turbulent kinetic energy at the mid-span transverse plane.
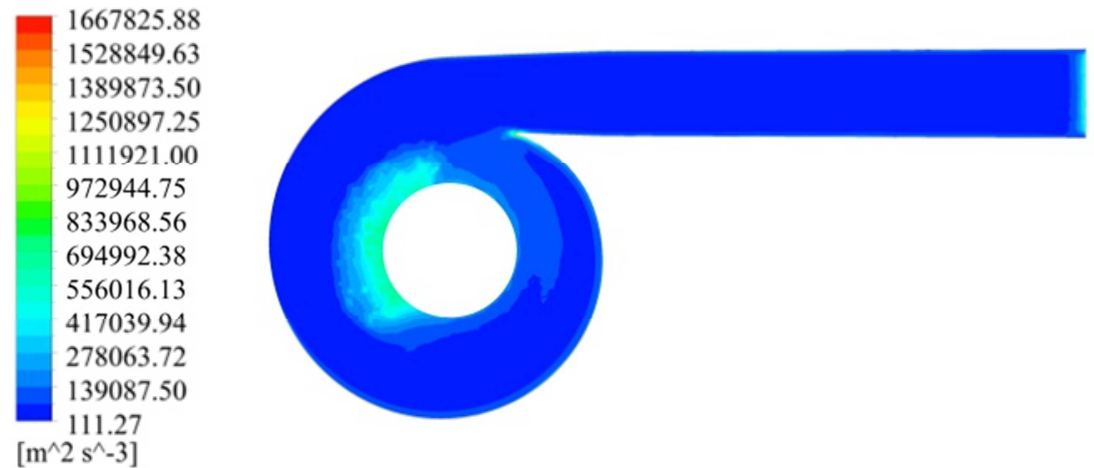

Figure 9. Distribution of the dissipation rate of the turbulent kinetic energy.

From these results, it is clear that the dissipation rate of the turbulent kinetic energy gets lower values at the volute inlet duct. Downstream of the volute inlet cross-section, it increases slightly along the volute scroll. Additionally, a wake zone characteristic of the maximum values of the dissipation rate of the turbulent kinetic energy has been recorded at the volute vane from $0^{\circ}$ to $135^{\circ}$ of the azimuthal angle. Furthermore, a rise of the dissipation rate of the turbulent kinetic energy has been detected at the volute tongue zone. These wake zones characteristic of the growth of the dissipation rate of the turbulent kinetic energy are eventually related to occurring losses which lead to reduce the work capacity of the flow.

\subsection{Turbulent Viscosity}

Figure 10 shows the distribution of the turbulent viscosity at the mid-span transverse plane. According to these results, it was apparent that the turbulent viscosity is quasi-null along the volute inlet duct. Then, from the volute inlet throat, it can be seen that the turbulent viscosity increases slowly through the volute scroll. However, a wake characteristic of the maximum values of the turbulent viscosity has been recorded at the volute near to the wall. Furthermore, this zone extends at the tongue upstream giving rise to an internal fluid friction at this zone.

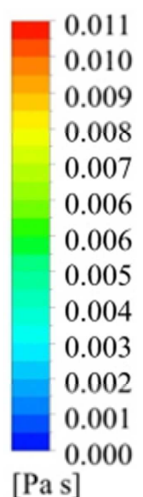

$[\mathrm{Pa} \mathrm{s}]$

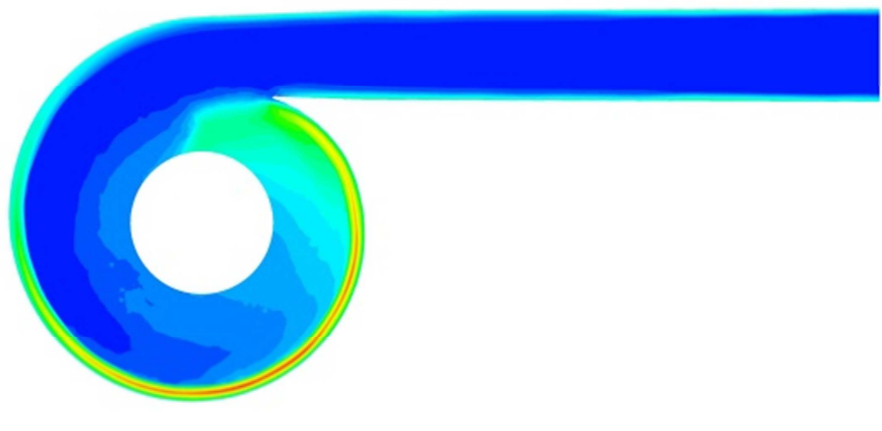

Figure 10. Distribution of the turbulent viscosity. 


\section{Conclusions}

This paper discusses the distribution of several flow parameters inside a vanned mixed flow turbine volute. The prediction of the flow field within the vanned volute is successfully obtained by means of our conducted simulations which are made by solving the Reynolds averaged Navier-Stokes equations. In fact, the reasonable agreement found between numerical and experimental results of the turbine overall performance ensures well our numerical model validity. Numerical results show that the flow changes its direction along the volute spiral and it leaves the volute vane with a high tangential component of the velocity. From the inlet duct to the vane exit, the static pressure drops significantly. Thus, the volute plays a vital role in the total reduction of the pressure within the full turbine stage. Besides, the flow within the volute can be considered uniform regarding that the production of the vorticity is limited to a small zone near the wall and the volute tongue. From $0^{\circ}$ to $135^{\circ}$ of the azimuthal angle, an excellent dissipation of the turbulent kinetic energy which yields eventually to occurring losses have been observed. Furthermore, upstream the volute tongue, there is an increase of the turbulent viscosity leading to an internal fluid friction.

\section{References}

[1] N. Lymberopoulos, N. C. Baines, N. Watson, Flow in single and twin-entry radial turbine volutes, ASME Paper 88-GT-59 (1988).

[2] F. Gu, A. Engeda, E. Benisek, A comparative study of incompressible and compressible design approaches of radial inflow turbine volutes, Proc Inst Mech Eng 215 (2001) 475-486.

[3] M. C. S. Barnard, R. S. Benson, Radial gaz turbines, Proc. IMechE 183 (1968) 59-70.

[4] Chen H, Design methods of volute casings for turbocharger turbine applications, J Power Energy 210 (1996) 149-156.
[5] Chen $\mathrm{H}$, A discussion on volute design method for radial inflow turbines, ASME paper GT2009-59110 (2009).

[6] A. Romagnoli, R. F. Martinez-Botas, Performance prediction of a nozzled and nozzleless mixed-flow turbine in steady conditions, International Journal of Mechanical Science 53 (2011) 557-574.

[7] M. Yang, R. Martinez Botas, S. Rajoo, T. Yokoyama, S. Ibaraki, Influence of volute cross-sectional shape of a nozzleless turbocharger turbine under pulsating flow condi-tions, Proceedings of ASME Turbo Expo 2014: GT2014-26150 (2014).

[8] M. Yang, R. Martinez Botas, S. Rajoo, T. Yokoyama, S. Ibaraki, An investigation of volute cross-sectional shape on turbocharger turbine under pulsating conditions in internal combustion engine, Energy Conversion and Management 105 (2015) 167-177.

[9] J. F. Suhrmann, D. Peitsch, M. Gugau, T. Heuer, On the effect of volute tongue design on radial turbine performance, ASME paper GT2012-69525 (2012).

[10] M. Abidat, M. K. Hamidou, M. Hachemi, M. Hamel, Design and flow analysis of radial and mixed flow turbine volutes, ASME paper GT2008-50503:2329-2338 (2008).

[11] S. Rajoo, Steady and pulsating performance of a variable geometry mixed flow turbochager turbine, $\mathrm{PhD}$ thesis, Imperial College, London, 2007.

[12] M. Abidat, Design and testing of a highly loaded mixed flow turbine. PhD thesis, Imperial College, London, 1991.

[13] Z. Driss, O. Mlayeh, S. Driss, D. Driss, M. Maaloul, M. S. Abid, Study of the incidence angle effect on the aerodynamic structure characteristics of an incurved Savonius wind rotor placed in a wind tunnel, Energy 894 (2016) 908-113.

[14] Z. Driss, O. Mlayeh, D. Driss, M. Maaloul, M. S. Abid, Numerical simulation and experimental validation of the turbulent flow around a small incurved Savonius wind rotor, Energy 74 (2014) 506-17.

[15] J. W. Lam, Q. Roberts, G. McDonnel, Flow modelling of a turbocharger turbine under pulsating flow, Preceedings of IMechE International Conference on Turbochargers and Turbocharging (2002) 181-197. 